\title{
Scientific Program
}

DAY1: Tuesday, October 11, 2016

SESSION 1: Studying Reactions at the Solid-Gas Interface with Environmental Electron Microscopy (Chair: Layla Mehdi)

- (invited) Marc Georg Willinger, "The Dynamics of Active Metal Catalysts Revealed by In Situ Electron Microscopy"

- (invited) Ovidiu Ersen, "Environmental TEM at Atmospheric Pressure: An Operando View of the Materials at the Nanoscale"

- Thierry Epicier, "Rapid Tomography in Environmental TEM: How Fast Can We Go to Follow the 3D Evolution of Nanomaterials In Situ?"

- Renaud Podor, "High Temperature and In Situ Study of SrO Surface Precipitation on Perovskite Ceramics"

SESSION 2: Liquid-Phase and Correlative Electron Microscopy of Soft Matter and Biominerals (Chair: Damien Alloyeau)

- (invited) Tanya Prozorov, "Correlative In Situ Analysis of Magnetosome Magnetite Biomineralization"

- Nico Sommerdijk, "Studying Polymer Self-Assembly by Combined Cryogenic and Liquid Phase Transmission Electron Microscopy"

- Anat Akiva, "Tracking the Early Events of Mineral Formation during Coral Development"

SESSION 3: In Situ Electron Microscopy of Electrochemical Processes (Chair: Kristian Mølhave)

- (keynote lecture) Frances M. Ross, "Liquid Cell Transmission Electron Microscopy for Imaging Electrochemical Processes"

- (invited) Chongmin Wang, "In-Situ S/TEM and In-Situ SIMS Multi-Modality Probing of Chemical and Structural Evolution of Rechargeable Battery"

- (invited) Layla Mehdi, "Understanding the Effect of Additives in Li-Ion and Li-Sulfur Batteries by Operando ec-(S)TEM"

- Walid Dachraoui, "In-Situ Liquid/Bias Transmission Electron Microscopy to Visualize the Electrochemical Lithiation/Delithiation Behaviors of LiFe0.5Mn0.5PO4"

\section{POSTER SESSION}

DAY2: Wednesday, October 12, 2016

SESSION 4: Liquid-Phase and Correlative Electron Microscopy of Cells and Proteins (Chair: Niels de Jonge)

- (invited) Diana Peckys, "Role of Heterogeneity in Cancer Cells Examined through Quantitative Analysis of Single HER2 Protein Distribution and Activation Status"

- (invited) Abraham (Bram) J. Koster, "Zooming in on Cells and Macromolecules with Correlative Light-Electron Electron Microscopy"

- (invited) Matthia Karreman, "Imaging Single Tumor Cells Invading Mouse Tissue using Multimodal Correlative Microscopy"

- Max Piffoux, "Monitoring Extracellular-Vesicles Dynamics at the Nanoscale by Liquid-Cell TEM" 
SESSION 5: Liquid-Phase Electron Microscopy of Nanomaterials (Chair: Nico Sommerdijk)

- (invited) Utkur Mirsaidov, "Visualizing Nanoscale Assembly in Solution using In Situ TEM"

- Arnaud Demortière, "Zig-Zag Self-Assembly of Magnetic Octahedral Fe3O4 Nanocrystals using In Situ Liquid Transmission Electron Microscopy"

- Nabeel Ahmad, "Shape Transformations during the Growth of Gold Nanostructures"

- Patricia Abellan, "Harnessing Control of Radiolysis during Liquid Cell Electron Microscopy to Enable Visualization of Nanomaterial Transformation Dynamics"

SESSION 6: Innovations in Methods for Correlative Microscopy (Chair: Thierry Epicier)

- (invited) Jacob Hoogenboom, "Super-Resolution Fluorescence in Electron Micrographs using InSitu Integrated Microscopy"

- Yutaro Yamada, "Correlative Atomic Force and Transmission Electron Microscopy toward Applications of Atomic Force Microscopy to Heterogeneous Systems"

- Christian Liebscher, "Topological Impurity Segregation at Faceted Silicon Grain Boundaries Studied by Correlative Atomic-Resolution STEM and APT"

SESSION 7: In Situ Measurements of Materials Properties (Chair: Kristian Mølhave)

- Karine Masenelli-Varlot, "Size and Environment Effect on the Room Temperature Plastic Deformation of Ceramic Nanoparticles"

- Sebastian Schmitt, "In-Situ Characterization of Individual Building Blocks for Nanophotonic Solar Cells by Correlative Microscopy"

- David Cooper, "Direct Observation of Redox Switching in Resistive Memory Devices Operated In-Situ in a Transmission Electron Microscope by Electron Energy Loss Spectroscopy and OffAxis Electron Holography"

POSTER SESSION (first author listed)

- Arne Janssen, "Real Time Evolution of the Reduction of Ilmenite in H2: A Correlative In Situ and Ex Situ Study"

- Christoph Luderer, "Ex-Situ Analysis and In-Situ Environmental TEM Studies of Manganite Perovskites for Catalytic Water Splitting"

- Siddardha Koneti, "Calcination of Pd Nanoparticles on Delta Alumina: Ex-situ Analysis versus In-situ Environmental TEM"

- Kristian Mølhave, "In-Situ TEM Investigation of Controlled VLS Silicon Nanowire Device Formation and Characterization"

- Renaud Podor, "In Situ Study of CeO2 Microspheres Sintering using HT-ESEM"

- Sergey V. Loginov, "Feasibility of Immuno-TRITC Labeling in Integrated 3D CLEM"

- Jantina Fokkema, "Fluorescently Labeled Silica Coated Metal Nanoparticles as Fiducial Markers for Correlative Light and Electron Microscopy"

- Hiroki Konno, "Direct Observation of Conformational Change of c-Cbl Ubiquitin Ligase by High Speed Atomic Force Microscopy"

- Tobias Caumanns, "In Situ and Cryo (S)TEM Imaging of Internal Microgel Architectures"

- Daniel J. Kelly, "Energy Dispersive X-Ray Spectroscopy in Liquids: Inorganic and Biological Applications" 
- Indra Navina Dahmke, "Electron Microscopy of Single Cells in Liquid for Stoichiometric Analysis of Transmembrane Proteins"

- Johan Salacroup, "CelDi: Development of an Advanced Solid / Fluid Reaction Stage for SEM"

- Andreas Hutzler, "Graphene-Supported Microwell Liquid Cell for In Situ Electron Microscopy in Materials Science"

- Juan Xiao, "Environmental Tomography of Liquid Latex Suspensions in STEM"

- Greg McMahon, "In Situ Characterization of ZnO Quantum Dots in Solution: Aggregation and Brownian Motion"

- Andreas Verch, "The Nucleation and Wetting Behavior at Hydrophilic, Polyacrylate Nanostructures Fabricated via Direct Laser Writing"

- Murat N. Yesibolati, "Using Microfluidic Chips with Nanochannels for Measuring the Mean Inner Potential of Liquid Water by Off-Axis Electron Holography"

Note: the program shown here reflects published abstracts and does not include abstracts of the corporate session nor withdrawn abstracts 